\title{
Antidepressivos, ansiolíticos, hipnóticos e sedativos: uma análise dos gastos em Minas Gerais
}

\author{
| ${ }^{1}$ Lucas Barbi, ${ }^{2}$ Liliany Mara Silva Carvalho, ${ }^{3}$ Tatiana Chama Borges Luz |
}

Resumo: A alocação de recursos com a aquisição de medicamentos é um dos maiores desafios para a efetivação da assistência farmacêutica no SUS. No entanto, poucos estudos avaliam a evolução desses gastos no nível estadual, especialmente com as classes dos antidepressivos, ansiolíticos e hipnótico-sedativos. O objetivo do estudo foi analisar e identificar os determinantes dos gastos com essas classes terapêuticas em Minas Gerais. Foram utilizados dados de compras públicas do Sistema Integrado de Administração de Materiais e Serviços (SIAD-MG) entre 2010 e 2015. Estimaram-se os gastos e volumes totais, além dos gastos com medicamentos náo constantes nas listas de medicamentos essenciais. As análises de “Top 10" e de "Drug Cost 90\%" identificaram os medicamentos responsáveis pelo maior gasto, e a análise de decomposição estabeleceu os determinantes da variação das despesas. Os gastos com as três classes totalizaram $\mathrm{R} \$ 81$ milhóes e aumentaram 2,5 vezes entre 2010 e 2015, passando de R \$ 7,5 milhóes para R \$ 18,7 milhões. Os antidepressivos representaram $89 \%$ dos gastos e $71 \%$ do volume adquirido. O preço foi o principal fator determinante do aumento das despesas, especialmente na classe dos antidepressivos. Os aumentos dos gastos ressaltam a necessidade de aprimoramento dos procedimentos de compra adotados pelo estado.

> Palavras-chave: Assistência Farmacêutica; gastos com medicamentos; antidepressivos, ansiolíticos, hipnóticos e sedativos.

\author{
1 Instituto René Rachou, Fundação \\ Oswaldo Cruz. Belo Horizonte- \\ MG, Brasil (lucas.costa@fiocruz.br). \\ ORCID: 0000-0002-5751-0445. \\ ${ }^{2}$ Instituto René Rachou, \\ Fundação Oswaldo Cruz. \\ Belo Horizonte-MG, Brasil \\ (lilianymara@bol.com.br). \\ ORCID: 0000-0003-0438-5174. \\ ${ }^{3}$ Instituto René Rachou, Fundação \\ Oswaldo Cruz. Belo Horizonte- \\ MG, Brasil (tatiana.luz@fiocruz.br). \\ ORCID: 0000-0003-1323-3105.
}

Recebido em: 16/11/2018 Revisado em: 02/07/2019 Aprovado em: 26/07/2019 


\section{Introdução}

Os medicamentos antidepressivos, ansiolíticos e hipnótico-sedativos fazem parte do grupo dos psicofármacos, sendo amplamente utilizados no mundo. $\mathrm{Na}$ Inglaterra, por exemplo, entre 1998 e 2010, o número de prescriçóes passou de mais de 34,7 milhôes para 59,8 milhôes, um crescimento de 72\% no período (ILYAS; MONCRIEFF, 2012). Já na Austrália, entre 2000 e 2011, esses medicamentos tiveram um aumento na dispensação de 59,6\% e representaram $83 \%$ do volume total de psicotrópicos dispensados no período (STEPHENSON; KARANGES; McGREGOR, 2013).

No Brasil, estudos sobre o uso desses medicamentos avaliam classes específicas, como, por exemplo, benzodiazepínicos ou de modo mais geral, englobando todos os psicofármacos, com estimativas de prevalências de uso que variam de 7,3\% a 25,8\%, a depender do subgrupo populacional avaliado (BORGES et al., 2015; ROCHA; WERLANG, 2013). Já estudos que avaliam o gasto são ainda mais restritos e não retratam especificamente essas classes. Uma investigação de âmbito nacional, de 2007 a 2014, mostrou um gasto federal de 54,6 milhôes de dólares com a aquisição de medicamentos do sistema nervoso central (MAGARINOS-TORRES et al., 2017).

Considerando-se que a demanda por medicamentos pela população tende a aumentar, um dos grandes motivos de preocupação no mundo, especialmente nos países com sistemas universais de saúde como o Brasil, é sua sustentabilidade financeira, uma vez que esses produtos podem chegar a consumir $60 \%$ dos orçamentos da saúde (BIGDELI et al., 2012).

Grande parte das compras públicas de medicamentos é feita no nível estadual. Além das compras de medicamentos sob sua pactuação, para órgãos que não fazem parte do SUS e das decorrentes da judicialização, estima-se que 65\% dos municípios brasileiros pactuaram a execução do orçamento da Assistência Farmacêutica de forma parcialmente ou totalmente centralizada nos estados, o que envolve a aquisição de medicamentos no referido nível (FALEIROS et al., 2017). A análise do perfil das aquisições públicas estaduais tem, então, um papel relevante para o aprimoramento dos procedimentos de planejamento e gestão da atividade.

O objetivo deste trabalho é avaliar as variaçôes nos gastos com medicamentos ansiolíticos, hipnótico-sedativos e antidepressivos adquiridos pelo Governo do Estado de Minas Gerais de 2010 a 2015, identificando os produtos responsáveis 
pelo maior gasto, bem como seus fatores determinantes, se preço, volume ou escolha terapêutica (ALVES et al., 2018; GERDTHAM; LUNDIN, 2004).

\section{Métodos}

Minas Gerais é um estado composto por 853 municípios, sendo o primeiro no Brasil em número de municípios e o segundo em população, com aproximadamente 21,1 milhóes de habitantes. O IDH é de 0,731 e corresponde à 9a colocação do país (IBGE, 2018a). Quanto ao perfil de morbidade em saúde mental, segundo a Pesquisa Nacional de Saúde, o estado ficou em $4^{\circ}$ lugar no país com relação à prevalência de depressáo em pessoas de 18 anos ou mais $(11,1 \%)$, sendo que destes, mais da metade $(54,4 \%)$ declarou usar algum medicamento para seu tratamento (IBGE, 2014).

A presente investigação está inserida no projeto "Estudo do abastecimento de medicamentos em Minas Gerais. Projeto Abastecer". Trata-se de um Estudo de Utilizaçâo de Medicamentos (EUM) longitudinal que objetiva investigar o abastecimento de medicamentos em Minas Gerais por meio da avaliação das compras públicas desses produtos. O presente trabalho possui foco específico nas compras de medicamentos ansiolíticos, antidepressivos, hipnótico-sedativos.

A fonte de dados é o Sistema Integrado de Administração de Materiais e Serviços (SIAD) do Estado de Minas Gerais, no período de 2010 a 2015, disponível sob demanda. O SIAD registra compras públicas e tem como objetivo integrar a gestão de compras à gestão orçamentária, sendo de uso obrigatório para a realização de licitaçôes pelos órgãos da administração pública (MINAS GERAIS, 2018). As variáveis pesquisadas foram os nomes dos medicamentos adquiridos com suas especificaçóes de princípio ativo, forma farmacêutica e concentração, o volume adquirido, informado de acordo com a unidade de aquisiçáo (comprimidos, ampolas, frascos etc.), o preço unitário de aquisição e o ano da compra.

Os medicamentos foram classificados segundo o Anathomical Therapeutical Chemical Classification System (ATC) e identificadas suas doses diárias definidas (DDDs). O ATC é um sistema de classificação internacional que subdivide os princípios ativos em grupos de acordo com sua ação nos órgãos e/ou sistemas e suas propriedades terapêuticas, farmacológicas e químicas (WHO, 2017). A DDD é conceituada como uma medida que sintetiza a dose média de manutenção diária 
para adultos considerando a indicação principal do medicamento (WHO, 2017).

Os medicamentos foram agregados segundo os subgrupos farmacológicos (ATC nível 3 - Ansiolíticos [N05B], Hipnóticos e Sedativos [N05C] e Antidepressivos [N06A]) para estimar os volumes adquiridos no período avaliado (anuais e total). $\mathrm{O}$ volume adquirido foi também agregado em DDDs. Para estimar esse volume, o número de unidades de cada medicamento foi multiplicado pela dosagem (concentração em miligramas) e dividido pelo valor da respectiva DDD. Para os fármacos em solução oral, o volume em DDDs foi padronizado por mililitros. A estimativa dos gastos foi realizada pela multiplicação do preço unitário dos produtos pelo volume de aquisiçáo. Os gastos foram estimados em reais $(\mathrm{R} \$)$, e os preços unitários de cada produto foram ajustados pela inflaçáo utilizando o Índice de Preços ao Consumidor Amplo (IPCA) em 31 de dezembro de 2015 (IBGE, 2018b). Por fim, cada medicamento foi classificado de acordo com sua presença nas listas oficiais de medicamentos essenciais, RENAME (Relação Nacional de Medicamentos Essenciais), nas versóes dos anos 2007, 2010, 2012 e 2014 (BRASIL, 2007, 2010, 2014, 2015) e RESME (Relação Estadual de Medicamentos Essenciais), nas versões dos anos 2009, 2010, 2011, 2012, 2013 e 2014, as quais foram solicitadas à Secretaria de Estado de Saúde de Minas Gerais (SES-MG).

Em primeiro lugar, foram estimados os gastos e volumes totais, segundo classe farmacológica e por DDD, e calculadas as respectivas variaçóes percentuais, tendo como referência os valores encontrados em 2010 em comparação aos valores encontrados para 2015. Adicionalmente, foram realizadas estimativas de gastos com medicamentos não constantes nas listas de medicamentos essenciais nos níveis nacional (RENAME) e estadual (RESME). O produto foi considerado ausente se náo constava nas respectivas listas vigentes no ano anterior à compra. Além dos medicamentos, foi verificado também o gasto com apresentaçôes farmacêuticas não inclusas.

Foram realizadas duas técnicas analíticas para identificar os medicamentos responsáveis pelo maior gasto: análise Top 10 e Drug Cost 90\%. A análise de Top 10 foi utilizada para verificar os dez medicamentos responsáveis pelo maior gasto, bem como a variação desse gasto entre 2010 e 2015. Para essa análise, os medicamentos foram organizados em ordem decrescente de gasto, tendo sido selecionados os dez fármacos, independentemente de sua apresentação farmacêutica, que representaram o maior gasto acumulado no período de 2010 a 2015. Para esses, foi avaliado o gasto em 2010 e em 2015 e estimada a variação correspondente. Foram estimados 
os gastos médios por DDD para o período (2010-2015), bem como o número total de DDDs adquiridas.

O método Drug Cost 90\% (DC90\%) foi utilizado para analisar os fármacos responsáveis por 90\% do gasto acumulado no período 2010 a 2015 (WETTERMARK et al., 2003). Os fármacos foram organizados em ordem decrescente de gasto no período, tendo sido estimados os valores totais em reais e em DDDs adquiridas. Em seguida, foram calculados os gastos percentuais por fármaco e acumulados.

Para avaliar os fatores que determinam a variação do gasto, foi utilizada a análise de decomposição (ALVES et al., 2018; GERDTHAM; LUNDIN, 2004), na qual calcula-se o indicador de variaçáo dos gastos (E), obtido a partir de três índices: a variação do preço $(\mathrm{P})$, a variação do volume $(\mathrm{V})$ e a escolha terapêutica $(\mathrm{D})$. Inicialmente, são estimados, para cada medicamento na apresentação farmacêutica correspondente, os preços médios ponderados e os volumes em DDDs no período base e no período de comparação (final). Em seguida, ainda para cada medicamento, são calculados os produtos entre preço médio no período base e volume no período base, preço médio no período base e volume no período final, e o preço médio do período atual e volume no período final. Por fim, os produtos estimados, bem como os volumes comprados em cada período, são agregados por classe farmacológica, para aplicação da seguinte fórmula:

$$
\mathrm{E}=\mathrm{P} \times \mathrm{V} \times \mathrm{D}=\frac{\sum \mathrm{P}_{1} \mathrm{~V}_{0}}{\sum \mathrm{P}_{0} \mathrm{~V}_{0}} \quad \mathrm{X} \quad \frac{\sum \mathrm{V}_{1}}{\sum \mathrm{V}_{0}} \quad \mathrm{X} \frac{\left(\sum \mathrm{P}_{1} \mathrm{~V}_{1} / \sum \mathrm{V}_{1}\right)}{\left(\sum \mathrm{P}_{1} \mathrm{~V}_{0} / \sum \mathrm{V}_{0}\right)}
$$

$\mathrm{P}_{0}=$ Preço médio ponderado por DDDs no período base

$\mathrm{P}_{1}=$ Preço médio ponderado por DDDs no período final

$\mathrm{V}_{0}=$ Volume comprado por apresentação farmacêutica em DDDs no período base

$\mathrm{V}_{1}=$ Volume comprado por apresentaçáo farmacêutica em DDDs no período final

Valores maiores do que 1 significam que o índice sob avaliação ( $\mathrm{P}, \mathrm{V}$ ou $\mathrm{D}$ ) contribui positivamente para a variação do gasto (E). Valores menores do que 1 significam que esse índice contribui negativamente para a variação do gasto e valores iguais a 1 mostram que o índice não contribui para a variação do gasto (GERDTHAM; LUNDIN, 2004).

As análises foram conduzidas por meio dos softwares Microsoft Excel 2016 (Microsoft Corporation, 2016) e IBM SPSS ${ }^{\oplus}$ Statistics. 


\section{Resultados}

Entre 2010 e 2015 foram gastos $\mathrm{R} \$ 81$ milhôes com a aquisição de mais de 1 bilhão de unidades (comprimidos, drágeas, cápsulas, ampolas e frascos) de medicamentos antidepressivos, ansiolíticos e hipnótico-sedativos em Minas Gerais. Foram identificados 37 medicamentos em 118 apresentaçóes farmacêuticas, os quais corresponderam a 10 subgrupos químicos (ATC nível 4). Foram adquiridas 720 milhōes de unidades em DDDs no período. Considerando-se somente os antidepressivos, foram identificados 20 medicamentos. Já os ansiolíticos compreenderam 9 medicamentos e os hipnótico-sedativos, 8 produtos.

Houve um aumento de 2,5 vezes nos gastos com as classes de medicamentos estudadas no período, passando de $\mathrm{R} \$ 7.459 .157,40$, em 2010 , para $\mathrm{R} \$ 18.735 .463,96$, em 2015. Particularmente, a classe dos ansiolíticos foi a que apresentou o maior aumento, de 22,4 vezes, passando de $\mathrm{R} \$ 59$ mil em 2010 para pouco mais de $\mathrm{R} \$$ 1,3 milhão, bem como a maior variação desse gasto no período, o que representou $2.135 \%$ (Tabela 1). 


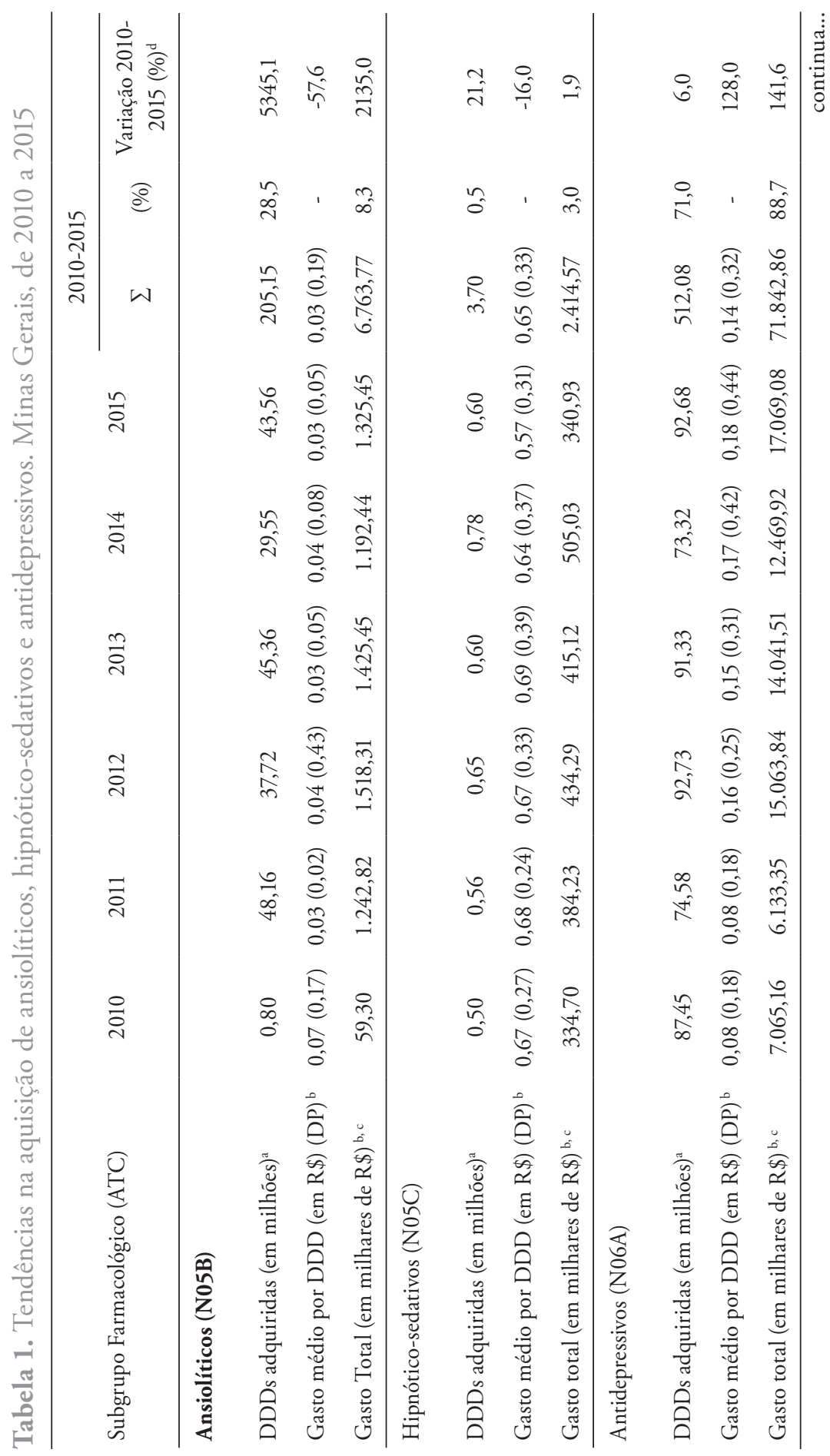


| Página 8 de 19

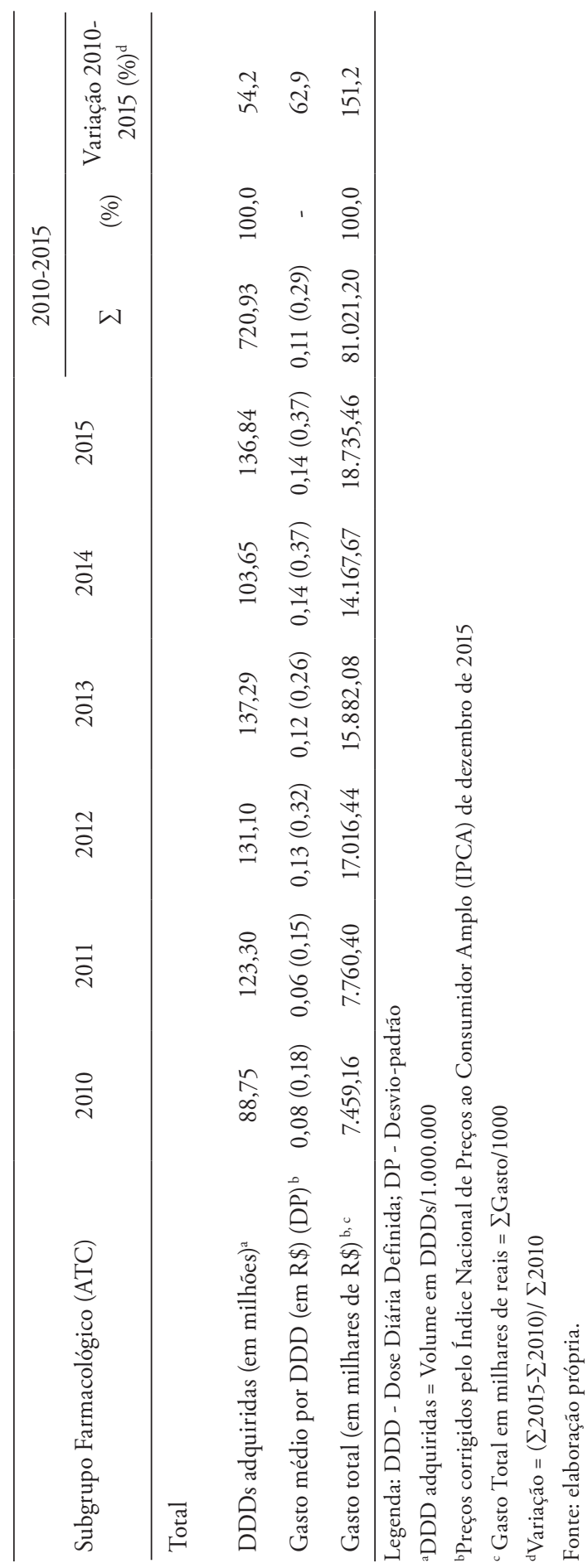


Em relação aos gastos por DDD adquirida, o valor médio estimado, considerando-se as três classes, foi de R $\$ 0,11$ para o período. Em 2012, esse valor chegou a R \$ 0,13 em comparaçáo a R \$ 0,06 em 2011, o que significou o maior aumento nos anos avaliados. O maior valor do gasto médio por DDD do período foi dos hipnótico-sedativos, $\mathrm{R} \$ 0,65$, e o menor registrado foi de $\mathrm{R} \$ 0,03$, para os ansiolíticos. Em relação à variação do gasto por $\mathrm{DDD}$, os ansiolíticos e os hipnóticosedativos apresentaram uma redução de 59,0\% e 16,0\%, respectivamente, enquanto os antidepressivos apresentaram um aumento de $128 \%$ nesse valor, registrando $\mathrm{R} \$$ 0,08 em 2010 e R \$ 0,18 em 2015 (tabela 1).

Avaliando-se a variaçáo dos gastos no período, percebe-se um grande salto das despesas de 2011 para 2012, aumentando 119,3\%. Já nos anos de 2013 e 2014, há uma pequena tendência de queda, mas esses gastos voltam a subir em 2015, chegando aos R \$ 18,7 milhóes (tabela 1). Com relação ao volume, por sua vez, verifica-se que houve um salto entre 2010 e 2011, com uma variação de 28,0\%, passando de 88,7 milhôes a 123,3 milhóes de DDDs. Entre 2012 e 2013, há um leve crescimento, apresentando queda em 2014, para em seguida, em 2015, voltar a subir (tabela 1).

Os antidepressivos foram responsáveis pelo maior gasto ( $\mathrm{R} \$ 71,8$ milhões ou $88,7 \%$ do total) e também pelo volume em DDDs (71,0\%). Em seguida, apareceram os ansiolíticos, que corresponderam a $8,3 \%$ (R $\$ 6,8$ milhóes) dos gastos e $28,5 \%$ das DDDs adquiridas. Os hipnótico-sedativos representaram 3\% dos gastos (R \$ 2,4 milhóes) e apenas $0,5 \%$ do volume adquirido em DDDs.

A tabela 2 apresenta os resultados do gasto com medicamentos antidepressivos, ansiolíticos e hipnótico-sedativos não incluídos nas listas de medicamentos essenciais. Considerando-se o padrão RENAME, durante o período analisado (2010-2015) foi gasto um total de $\mathrm{R}$ \$ 8,6 milhóes para aquisição de 29 medicamentos que não integravam essa relação, correspondendo a $10,6 \%$ do total de gastos. Esses gastos passaram de R \$ 629 mil em 2010 para R \$ 2,7 milhóes em 2015, um aumento de 4,5 vezes. Considerando-se o padrão RESME, os gastos efetuados com medicamentos não constantes dessas listas, o total foi de $\mathrm{R}$ \$ 5,6 milhôes no período (6,9\% das despesas totais), que foi destinado à aquisição de 30 medicamentos. Esses gastos passaram de R \$ 564 mil em 2010 para R \$ 1,1 milhão em 2015, representando um aumento de quase duas vezes no período (tabela 2). 
Tabela 2. Gastos com medicamentos ausentes das listas de medicamentos essenciais, estadual e nacional. Minas Gerais, de 2010 a 2015

\begin{tabular}{|c|c|c|c|c|c|c|}
\hline \multirow{3}{*}{$\begin{array}{l}\text { Subgrupo Farmacológico } \\
\text { (ATC) }\end{array}$} & \multicolumn{6}{|c|}{ Valor total (em milhares de $\mathrm{R} \$)^{\mathrm{a}, \mathrm{b}}$} \\
\hline & \multicolumn{2}{|c|}{2010} & \multicolumn{2}{|c|}{2015} & \multicolumn{2}{|c|}{ Período (2010-2015) } \\
\hline & $\begin{array}{c}\text { RESME } \\
\text { (2009) }\end{array}$ & $\begin{array}{c}\text { RENAME } \\
(2007)\end{array}$ & $\begin{array}{c}\text { RESME } \\
\text { (2014) }\end{array}$ & $\begin{array}{c}\text { RENAME } \\
\text { (2014) }\end{array}$ & RESME & RENAME \\
\hline Ansiolíticos (N05B) & 42,18 & 20,02 & 36,59 & 36,59 & 266,09 & 243,94 \\
\hline Hipnótico-sedativos (N05C) & 334,70 & 7,3 & 340,93 & 17,29 & $2.414,57$ & 84,25 \\
\hline Antidepressivos (N06A) & 187,37 & 602,03 & 692,41 & $2.728,36$ & $2.939,25$ & $8.276,22$ \\
\hline$\sum$ Total & 564,25 & 629,35 & $1.069,93$ & $2.782,24$ & $5.619,91$ & $8.604,41$ \\
\hline Proporção do gasto $(\%)^{\mathrm{c}}$ & 7,6 & 8,4 & 5,7 & 14,6 & 6,9 & 10,6 \\
\hline
\end{tabular}

${ }^{a}$ Preços corrigidos pelo Índice Nacional de Preços ao Consumidor Amplo (IPCA) de dezembro de 2015

${ }^{\mathrm{b}}$ Gasto total em milhares de reais= $\sum$ Gasto $/ 1000$

c Proporçấo do gasto $=\sum$ Gasto com medicamentos ausentes das listas/Gasto anual total

Fonte: elaboração própria.

Adicionalmente, a avaliação dos gastos efetuados com apresentações farmacêuticas que não constavam nas referidas listas de medicamentos mostrou que as despesas, no período, foram da ordem de R \$ 16,4 milhóes, considerando-se a RESME (20,2\% do total gasto), e de R \$ 18,4 milhóes, considerando-se a RENAME (23,0\% do valor total gasto).

A tabela 3 apresenta o resultado da análise Top 10. Os fármacos que tiveram maior variaçáo de gastos no período foram os ansiolíticos Diazepam (6.485\%) e Clobazam (628\%), seguidos dos antidepressivos Amitriptilina (546\%) e Imipramina (412\%). Quando considerado os gastos por DDD, os medicamentos mais caros são os antidepressivos Duloxetina e Venlafaxina, com valores registrados de R \$ 6,99 e de $\mathrm{R} \$ 2,64$, respectivamente. Os medicamentos com menor valor por DDD foram Diazepam $(\mathrm{R} \$ 0,03)$ e a Fluoxetina $(\mathrm{R} \$ 0,05)$. 


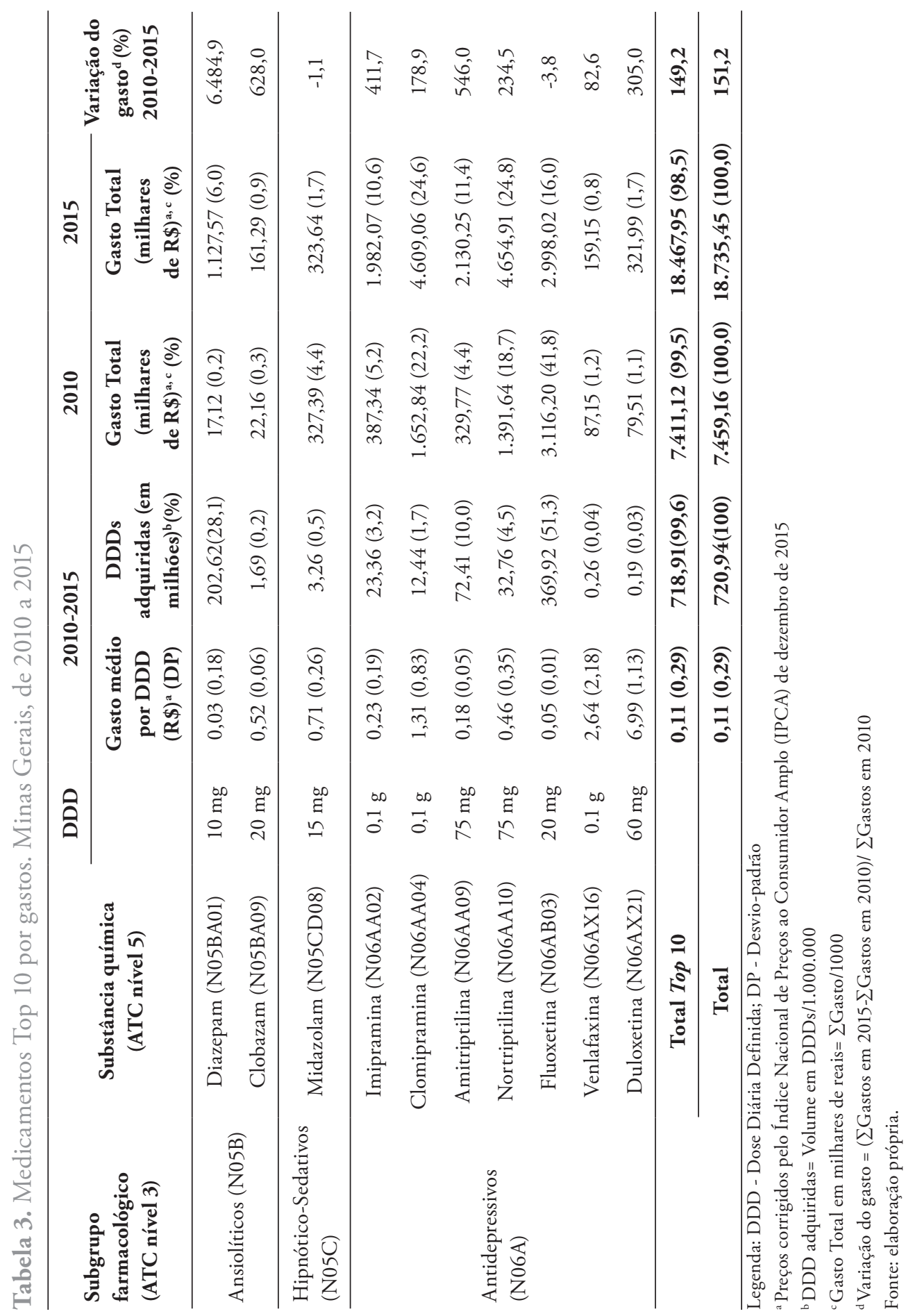


Os resultados da análise de Drug Cost 90\% (DC90\%) estão apresentados na Tabela 4. Seis medicamentos (Fluoxetina, Clomipramina, Nortriptilina, Amitriptilina, Diazepam e Imipramina) corresponderam a mais de $90 \%$ do gasto, compreendendo $\mathrm{R} \$ 74,5$ milhóes no período analisado, estando todos eles presentes nas listas estaduais e nacionais do período entre 2010 e 2015 (tabela 4).

Os resultados da análise de decomposição estão apresentados na tabela 5. Em termos gerais, a variaçáo de preço $(\mathrm{P}=1,91)$ e de volume $(\mathrm{V}=1,54)$ afetaram positivamente o aumento da despesa $(E=2,54)$, e a escolha terapêutica afetou negativamente $(D=0,86)$. No caso dos ansiolíticos, o principal determinante do aumento do gasto no período foi o volume $(V=54,5)$. Para os hipnótico-sedativos, a variaçáo do volume seguida da escolha terapêutica foram os fatores determinantes do gasto. Para essa classe, a variação de preço contribuiu negativamente para o gasto. Finalmente, para a classe dos antidepressivos, os três fatores contribuíram positivamente para o aumento do gasto, sendo o preço o principal desses fatores $(\mathrm{P}=1,97)$.

Tabela 4. Análise de drug cost (DC 90\%). Minas Gerais, de 2010 a 2015

\begin{tabular}{|c|c|c|c|c|}
\hline & Substância Química (ATC) & Gasto $(\mathbf{R} \$)^{\mathrm{a}}$ & $\begin{array}{c}\text { Gasto do } \\
\text { período }(\%)\end{array}$ & $\begin{array}{c}\text { Gasto } \\
\text { acumulado (\%) }\end{array}$ \\
\hline 1 & Fluoxetina (N06AB03) & $19.373 .750,60$ & 23,9 & 23,9 \\
\hline 2 & Clomipramina (N06AA04) & $16.324 .553,05$ & 20,1 & 44,0 \\
\hline 3 & Nortriptilina (N06AA10) & $15.071 .327,70$ & 18,6 & 62,6 \\
\hline 4 & Amitriptilina (N06AA09) & $12.783 .878,04$ & 15,8 & 78,4 \\
\hline 5 & Diazepam (N05BA01) & $5.647 .374,03$ & 7,0 & 85,4 \\
\hline 6 & Imipramina (N06AA02) & $5.336 .964,48$ & 6,6 & 92,0 \\
\hline \multirow[t]{2}{*}{7} & Outros & $6.483 .354,49$ & 8,0 & 100 \\
\hline & Total & 81.021.202,39 & 100,0 & \\
\hline
\end{tabular}

${ }^{\text {a }}$ Preços corrigidos pelo Índice Nacional de Preços ao Consumidor Amplo (IPCA) de dezembro de 2015 Fonte: elaboração própria. 
Tabela 5. Análise de decomposição total e por subgrupo farmacológico das compras de ansiolíticos, hipnótico-sedativos e antidepressivos. Minas Gerais, de 2010 a 2015

\begin{tabular}{lcccc}
\hline $\begin{array}{l}\text { Subgrupo farmacológico } \\
\text { (ATC) }\end{array}$ & Preço (P) $^{\mathbf{a}}$ & Volume (V) $^{\mathbf{b}}$ & $\begin{array}{c}\text { Escolha terapêutica } \\
(\mathbf{D})^{\mathbf{c}}\end{array}$ & Gasto (E) $^{\mathbf{d}}$ \\
\hline Ansiolíticos (N05C) & 1,11 & 54,45 & 0,43 & 26,26 \\
Hipnótico-sedativos (N05B) & 0,80 & 1,21 & 1,05 & 1,02 \\
Antidepressivos (N06A) & 1,97 & 1,06 & 1,16 & 2,42 \\
\hline Todas as classes & $\mathbf{1 , 9 1}$ & $\mathbf{1 , 5 4}$ & $\mathbf{0 , 8 6}$ & 2,54 \\
\hline
\end{tabular}

${ }^{\mathrm{a}} P=\frac{\sum P_{1} Q_{0}}{\sum P_{0} Q_{0}}{ }^{\mathrm{b}} V=\frac{\sum V_{1}}{\sum \mathrm{V}_{0}}{ }^{\mathrm{c}} D=\frac{\left(\sum P_{1} Q_{1} / \sum Q_{1}\right)}{\left(\sum P_{1} Q_{0} / \sum Q_{0}\right)}{ }^{\mathrm{d}} E=P \times V \times D$

Fonte: elaboração própria.

\section{Discussão}

Este trabalho é pioneiro na análise do gasto público com medicamentos antidepressivos, ansiolíticos e hipnótico-sedativos em um importante estado brasileiro, Minas Gerais, o primeiro do país em número de municípios, o segundo em população e o terceiro em participação no Produto Interno Bruto (PIB) nacional (IBGE, 2017, 2018a). No período analisado, foram gastos $\mathrm{R} \$ 81$ milhóes com a aquisição dessas classes de medicamentos. As análises sugerem que, de modo geral, houve opção por escolhas terapêuticas mais baratas; porém, houve aumentos tanto do preço quanto do volume adquirido no período, o que acarretou um aumento geral do gasto.

Dentre as três classes analisadas, os principais responsáveis pelo gasto e pelo volume adquirido foram os medicamentos antidepressivos, com $88,7 \%$ e $71 \%$ dos totais, respectivamente. Houve, ainda, um aumento médio no gasto por DDD de $128 \%$. Sabe-se que esses medicamentos são amplamente utilizados no mundo, tendo seu consumo dobrado nos países da Organização para a Cooperação e Desenvolvimento Econômico (OCDE) entre 2000 e 2015(OECD, 2017). Mars et al. (2017) explicam o aumento do consumo de antidepressivos com base na melhoria dos critérios diagnósticos das condiçôes passíveis de tratamento com esses medicamentos, na maior disponibilidade de opçôes terapêuticas e também em funçáo das atitudes dos prescritores, que estariam prescrevendo mais esses tipos de medicamentos a seus pacientes. 
De fato, cinco dos seis principais medicamentos responsáveis pelo maior gasto são da classe dos antidepressivos, com destaque para a Fluoxetina, que consumiu quase um quarto dos recursos no período analisado. Esse medicamento, em particular, tem sido o antidepressivo mais prescrito no mundo desde seu lançamento em 1988 (BARAKAT; HAMDY; ELBADR, 2018). Por outro lado, dados internacionais sobre a despesa com antidepressivos apontam para um comportamento oposto aos resultados desta investigação. Estudo comparativo realizado entre 10 países desenvolvidos mostrou que o gasto com antidepressivos em sistemas universais de saúde tendem a ser menores (MORGAN; LEOPOLD; WAGNER, 2017). Na Espanha foi registrada uma redução de $29 \%$ no custo da DDD dos antidepressivos entre 2000 e 2011 (VERDÚ et al., 2014). Estudo britânico apontou uma reduçáo de 62,4\% dos gastos com antidepressivos entre 1998 e 2010(ILYAS; MONCRIEFF, 2012).

Apesar de não haver dados que permitam estimar a necessidade de antidepressivos pela população em Minas Gerais, autores mostram que o estado aparece em quarto lugar no país em prevalência de autorrelato de diagnóstico de depressão em adultos $(11,1 \%)$ e que essa prevalência é, ainda, cerca de $50 \%$ maior do que os valores calculados para o país como um todo (STOPA et al., 2015). Por outro lado, a literatura aponta que adultos e idosos com depressão e que obtêm medicamentos exclusivamente pelo SUS compreendem apenas cerca de 30\% a 40\% desse grupo populacional (LOPES et al., 2016; MATTA et al., 2018). Dessa forma, dado que o volume de antidepressivos não apresentou aumento expressivo ao longo do período observado, pode-se supor que o volume adquirido seja insuficiente para o atendimento da demanda por esses medicamentos.

Com relação aos ansiolíticos e hipnótico-sedativos, é importante ressaltar que eles tiveram um gasto bem menos expressivo em comparação aos antidepressivos. Os ansiolíticos, em particular, foram a classe que apresentou a maior variação na despesa, de 26,2 vezes, e do volume, de 54,5 vezes, entre 2010 e 2015, apesar de haver uma redução de 57,6\% nos gastos médios por DDD. No caso dessa classe, o volume foi o maior responsável pela variação da despesa observada e esses aumentos podem ser explicados pela inclusão de fármacos ansiolíticos nas listas de medicamentos essenciais de Minas Gerais a partir de 2009 (MINAS GERAIS, 2009).

Dentre os hipnótico-sedativos, o único fármaco de destaque é o Midazolam, utilizado na terapêutica para o tratamento de insônia e também como anticonvulsivo e adjuvante anestésico (BRASIL, 2007). Os resultados encontrados para essas 
classes vão ao encontro de estudos internacionais, que mostram reduções também significativas nos gastos médios por DDD com ansiolíticos e hipnótico-sedativos (MACHADO-ALBA; ALZATE-CARVAJAL; JIMENEZ-CANIZALES, 2015; VERDÚ et al., 2014).

No Brasil, são escassas as informações sobre consumo e gasto com ansiolíticos e hipnótico-sedativos. No entanto, investigação de base populacional nacional no setor privado mostrou um aumento de $72 \%$ no consumo de benzodiazepínicos entre 2010 e 2012, variando de 2,63 a 4,53 DHD (AZEVEDO; ARAÚJO; FERREIRA, 2016). É possível que o sistema público esteja acompanhando essa tendência, passando a ofertar mais medicamentos dessas classes para a população.

A análise dos gastos com medicamentos não constantes nas listas de medicamentos essenciais (RENAMEs e RESMEs) revelou despesas da ordem de 10,6\% e de 6,9\% do total, respectivamente. Apesar de essas despesas serem relativamente reduzidas quando comparadas aos gastos totais, é importante que se avalie em até que ponto as listas de medicamentos essenciais estão, de fato, sendo utilizadas como norteadoras das compras públicas. Magarinos-Torres et al. (2017), em uma avaliação nacional da implementação da RENAME entre 2007 e 2014, encontraram gastos bem mais expressivos com medicamentos não inclusos, da ordem de 60,5\% do total (US\$ 9,6 bilhōes) (MAGARINOS-TORRES et al., 2017). Entretanto, é necessário levar em conta que os dados daqueles autores refletiram compras que abrangeram órgãos que não integram o SUS, dificultando a comparação direta dos resultados encontrados nessa investigação.

Este estudo permite o entendimento claro com relação às despesas com antidepressivos, ansiolíticos e hipnótico-sedativos. Foram estimados os valores gastos e delineado um perfil detalhado das classes terapêuticas adquiridas, seu gasto médio por DDD, a variação dos gastos, bem como estabelecidos quais foram os fatores determinantes do gasto. Algumas limitaçóes merecem consideração. A fonte de dados reflete a aquisição dos medicamentos pelo estado, não sendo possível estabelecer uma relação direta entre o que foi adquirido e o que foi, de fato, consumido pela população, sendo as estimativas de volume apresentadas consideradas um proxy desse consumo (LUZ et al., 2017). Desse modo, não é possível determinar se o aumento de volume observado é resultado do aumento da cobertura populacional para esses medicamentos no período avaliado. Por outro lado, a base de dados utilizada apresenta diversas vantagens para sua utilização em estudos dessa natureza, 
com boa representatividade e cobertura das compras, confiabilidade e padronização dos dados coletados e continuidade no registro desses dados ao longo do tempo, permitindo comparaçôes longitudinais.

\section{Considerações finais}

O aumento de gasto com antidepressivos merece uma atenção especial. Enquanto o aumento no volume, apesar de pequeno, se alinha com o que estudos internacionais têm verificado, o nível dos gastos caminha em direção oposta, tendo crescido significativamente. De fato, a análise de decomposição mostra que o preço é o principal responsável pela variaçáo dos gastos observada com esses medicamentos. Esses resultados parecem indicar que é necessário um maior cuidado na realização dos processos licitatórios desses produtos, para identificar possível sobrepreço.

Os resultados apresentados podem fornecer subsídios importantes para o aprimoramento das compras públicas estaduais que ensejam o fortalecimento do uso dos critérios de essencialidade nas aquisiçôes. Para os tomadores de decisão, esses resultados podem contribuir para a melhoria da gestão e otimização dos recursos financeiros disponíveis.

De forma complementar, as estimativas dos gastos com antidepressivos, ansiolíticos e hipnótico-sedativos podem auxiliar nas pesquisas em saúde mental, permitindo comparação dos valores estimados com as despesas realizadas com outras açôes de saúde na Rede de Atenção Psicossocial (RAPS). ${ }^{1}$

\section{Referências}

ALVES, J. C. et al. Immunosuppressants in Brazil: underlying drivers of spending trends, 20102015. Expert Review of Pharmacoeconomics \& Outcomes Research, v. 18, n. 5, p. 1-8, 14 jun. 2018. AZEVEDO, A. J. P. de; ARAÚJO, A. A.; FERREIRA, M. A. F. Consumo de ansiolíticos benzodiazepínicos: uma correlação entre dados do SNGPC e indicadores sociodemográficos nas capitais brasileiras. Ciência \& Saúde Coletiva, v. 21, n. 1, p. 83-90, 2016.

BARAKAT, A.; HAMDY, M. M.; ELBADR, M. M. Uses of fluoxetine in nociceptive pain management: A literature overview. European Journal of Pharmacology, v. 829, p. 12-25, jun. 2018.

BIGDELI, M. et al. Access to medicines from a health system perspective. Health Policy and Planning, v. 28, n. 7, p. 692-704, nov. 2012.

BORGES, T. L. et al. Prevalência do uso de psicotrópicos e fatores associados na atenção primária à saúde. Acta Paulista de Enfermagem, v. 28, n. 4, p. 344-349, ago. 2015. 
BRASIL. Ministério da Saúde. Secretaria de Ciência, Tecnologia e Insumos Estratégicos. Departamento de Assistência Farmacêutica e Insumos Estratégicos. Relação Nacional de Medicamentos Essenciais: RENAME. Brasília, DF: Ministério da Saúde, 2007.

- Ministério da Saúde. Secretaria de Ciência, Tecnologia e Insumos Estratégicos. Departamento de Assistência Farmacêutica e Insumos Estratégicos. Relaçâo Nacional de Medicamentos Essenciais: RENAME 2010. Brasília, DF: Ministério da Saúde, 2010.

. Ministério da Saúde. Secretaria de Ciência, Tecnologia e Insumos Estratégicos. Departamento de Assistência Farmacêutica e Insumos Estratégicos. Relação Nacional de Medicamentos Essenciais: RENAME 2013. Brasília, DF: Ministério da Saúde, 2014.

. Ministério da Saúde. Secretaria de Ciência, Tecnologia e Insumos Estratégicos. Departamento de Assistência Farmacêutica e Insumos Estratégicos. Relação Nacional de Medicamentos Essenciais: RENAME 2014. Brasília, DF: Ministério da Saúde, 2015.

FALEIROS, D. R. et al. Financing of Pharmaceutical Services in the municipal management of the Brazilian Unified Health System. Revista de Saúde Pública, v. 51, n. supl.2, nov. 2017.

GERDTHAM, U-G.; LUNDIN, D. Why Did Drug Spending Increase During the 1990s? A Decomposition Based on Swedish Data. PharmacoEconomics, v. 22, n. 1, p. 29-42, jan. 2004.

INSTITUTO BRASILEIRO DE GEOGRAFIA E ESTATÍSTICA. IBGE Cidades. Disponível em: <https://cidades.ibge.gov.br/brasil>. Acesso em: 25 jun. 2018a.

. Indice Nacional de Preços ao Consumidor Amplo: IBGE. . Acesso em: 6 abr. 2018b.

. (Org.). Pesquisa nacional de saúde, 2013: percepçáo do estado de saúde, estilos de vida

e doenças crônicas: Brasil, grandes regióes e unidades da Federação. Rio de Janeiro: Instituto Brasileiro de Geografia e Estatística, 2014.

. Sistema de Contas Regionais: Brasil. Rio de Janeiro-RJ: Instituto Brasileiro de Geografia e Estatística, 2017.

ILYAS, S.; MONCRIEFF, J. Trends in prescriptions and costs of drugs for mental disorders in England, 1998-2010. British Journal of Psychiatry, v. 200, n. 5, p. 393-398, 2012.

LOPES, C. S. et al. Inequities in access to depression treatment: results of the Brazilian National Health Survey - PNS. International Journal for Equity in Health, v. 15, n. 1, dez. 2016. Disponível em: <http://equityhealthj.biomedcentral.com/articles/10.1186/s12939-016-04461>. Acesso em: 30 jun. 2019.

LUZ, T. C. B. et al. Trends in medicines procurement by the Brazilian federal government from 2006 to 2013. PLOS ONE, v. 12, n. 4, p. e0174616, abr. 2017.

MACHADO-ALBA, J. E.; ALZATE-CARVAJAL, V.; JIMENEZ-CANIZALES, C. E. Tendencias de consumo de medicamentos ansiolíticos e hipnóticos en una población colombiana, 2008-2013. Revista Colombiana de Psiquiatría, v. 44, n. 2, p. 93-99, jun. 2015. 
MAGARINOS-TORRES, R. et al. Essential medicines list implementation dynamics: a case study using Brazilian federal medicines expenditures. Basic \& Clinical Pharmacology \& Toxicology, v. 121, n. 3, p. 181-188, maio 2017.

MARS, B. et al. Influences on antidepressant prescribing trends in the UK: 1995-2011. Social Psychiatry and Psychiatric Epidemiology, v. 52, n. 2, p. 193-200, fev. 2017.

MATTA, S. R. et al. Fontes de obtenção de medicamentos por pacientes diagnosticados com doenças crônicas, usuários do Sistema Único de Saúde. Cadernos de Saúde Pública, v. 34, n. 3, p. e00073817, abr. 2018.

MINAS GERAIS. Secretaria de Estado de Planejamento e Gestão. Portal de Compras do Estado de Minas Gerais. Disponível em: <http://www.compras.mg.gov.br>. Acesso em: 10 abr. 2018.

Secretaria de Estado de Saúde. Relação de Medicamentos do Estado de Minas Gerais. Belo Horizonte, MG: Superintendência de Assistência Farmacêutica, 2009.

MORGAN, S. G.; LEOPOLD, C.; WAGNER, A. K. Drivers of expenditure on primary care prescription drugs in 10 high-income countries with universal health coverage. Canadian Medical Association Journal, v. 189, n. 23, p. E794-E799, jun. 2017.

ORGANIZAÇÃO PARA COOPERAÇÃO E DESENVOLVIMENTO ECONÔMICO (Org.). Health at a glance 2017: OECD indicators. Paris, France: OECD, 2017.

ROCHA, B. S.; WERLANG, M. C. Psicofármacos na Estratégia Saúde da Família: perfil de utilização, acesso e estratégias para a promoção do uso racional. Ciência \& Saúde Coletiva, v. 18, n. 11, p. 3291-3300, nov. 2013.

STEPHENSON, C. P.; KARANGES, E.; MCGREGOR, I. S. Trends in the utilisation of psychotropic medications in Australia from 2000 to 2011. Australian \& New Zealand Journal of Psychiatry, v. 47, n. 1, p. 74-87, jan. 2013.

STOPA, S. R. et al. Prevalência do autorrelato de depressão no Brasil: resultados da Pesquisa Nacional de Saúde, 2013. Revista Brasileira de Epidemiologia, v. 18, n. supl 2, p. 170-180, dez. 2015.

VERDÚ, E. S. et al. Evolución de la utilización de antidepresivos, ansiolíticos e hipnóticos en la Comunitat Valenciana. Período 2000-2010. Atención Primaria, v. 46, n. 8, p. 416-425, out. 2014.

WETTERMARK, B. et al.Drug utilisation 90\% profiles:a useful tool for quality assessment of prescribing in primary health care in Stockholm. Pharmacoepidemiology and Drug Safety, v. 12, n. 6, p. 499-510, set. 2003.

WORLD HEALTH ORGANIZATION. Guidelines for ATC Classification and DDD assignment 2018. Oslo, Noruega: World Health Organization, 2017. Acesso em: 2 ago. 2018.

\section{Nota}

${ }^{1}$ L. Barbi, L. M. S. Carvalho e T. C. B Luz foram responsáveis pela concepção, planejamento, análise, interpretação dos dados, redação e aprovação da versão final do artigo. 


\section{Abstract}

Antidepressive, anti-anxiety and hypnotics
and sedatives agents: an analysis of public
expenditure in Minas Gerais state, Brazil The allocation of financial resources on medicines procurement is one of the greatest challenges to the effectiveness of the Pharmaceutical Services in the Brazilian National Health System. However, there are few studies evaluating this expenditure trends at state level, especially with antidepressants, anxiolytics, hypnotics and sedatives. The study evaluated public expenditure trends and drivers for these therapeutic classes in Minas Gerais state, Brazil, by using data from SIAD database [Sistema Integrado de Administração de Materiais e Serviços] from 2010 to 2015. Total expenditure, volume and also expenditures with medicines not included in the essential lists were estimated. The top 10 medicines in terms of expenditure and drugs accounting for $90 \%$ of the total cost were identified. Decomposition analysis was conducted to assess the drivers of expenditure. Expenditure in the period totaled $\mathrm{R} \$ 81$ million and increased 2.5 times from 2010 to 2015. Antidepressants accounted for $89 \%$ of expenses and $71 \%$ of volume. Price contributed positively to the expenditure variation during the period, especially in antidepressants. The results highlight the need of improvement of public procurement procedures adopted by Minas Gerais state.

> Keywords: pharmaceutical services; drug costs; antidepressive, anti-anxiety and hypnotics and sedatives agents. 